\title{
Treatment of Glycine max seeds with gibberellins alters root morphology, anatomy, and transcriptional networks
}

\author{
Y.Q. HAN ${ }^{1,2}$, Y. SHI ${ }^{3}$, Y.M. GAO 1 , J.D. DU ${ }^{4}$, N.J. FENG ${ }^{4}$, Y.X. ZHANG ${ }^{4}$, and D.F. ZHENG ${ }^{2,5 *}$ \\ College of Life Science and Technology, Heilongjiang Bayi Agricultural University, \\ Daqing, Heilongjiang 163319, P.R. China ${ }^{1}$ \\ National Coarse Cereals Engineering Research Center, Daqing, Heilongjiang 163319, P.R. China ${ }^{2}$ \\ Fu Dekang Economic and Trade Development Co., Beijing, 100031, P.R. China ${ }^{3}$ \\ College of Agriculture, Heilongjiang Bayi Agricultural University, Daqing, Heilongjiang 163319, P.R. China \\ College of Agriculture, Guangdong Ocean University, Zhanjiang, Guangdong 524088, P.R. China ${ }^{5}$
}

\begin{abstract}
Gibberellins (GAs) regulate diverse aspects of growth and development, but their role in root development and lateral root (LR) formation is poorly understood. In this study, $\mathrm{GA}_{3}$ was applied to soybean [Glycine max (L.) Merr] by seed soaking. The results showed that root length and root surface area were significantly inhibited in early stages after GA $\mathrm{G}_{3}$ treatment. Microscopic examination showed that $\mathrm{GA}_{3}$ treatment changed the cortex thickness, the pericycle diameter, and cell size in main root. Interestingly, exogenous $\mathrm{GA}_{3}$ increased the quantity of lateral root primordia (LRP), but LR number decreased in this period. Moreover, the content of GAs, auxin and abscisic acid in root was altered. RNA-seq results revealed that application of $\mathrm{GA}_{3}$ not only changed the expression of genes in GA biosynthesis pathway, including GA20ox and GA2ox, but also the GA regulation genes and signalling pathway genes. The changes in expression of gene concerning other hormones were also detected. In addition, $\mathrm{GA}_{3}$ altered cell wall biogenesis and degradation genes which might be related to the changes of root morphology. In response to increased $\mathrm{GA}_{3}, 103$ transcription factors were detected. Thus, exogenous $\mathrm{GA}_{3}$ changed the content of hormones in roots and affected the root development by regulating the expression of respective genes.
\end{abstract}

Additional key words: gene expression, lateral root, main root, RNA-seq, seed soaking, soybean.

\section{Introduction}

Soybean [Glycine $\max (\mathrm{L}$.$) Merr] is an important crop. A$ taproot-type soybean root system, consists of an embryoderived main root (MR) and the lateral roots (LRs) which are continuously produced from MR. LRs are the most vigorous and physiologically active part of the soybean root system which is required for crop development, yield, and quality. Because of its significance, the mechanism of root development and LR formation have been intensively studied (Osmont et al. 2007, Slovak et al. 2015). The mechanism of root development and LR formation involves complex gene regulatory networks in model plant Arabidopsis. However, the detailed gene network mechanism of root development is largely unclarified in soybean. Among the studies related to soybean root, many studies focus on the response of root to biotic and abiotic stresses (Radwan et al. 2011, Song et al. 2016).

\footnotetext{
Submitted 30 March 2019, last revision 24 August 2019, accepted 30 September 2019.

Abbreviations: ABA - abscisic acid; CKs - cytokinins; DEG - differentially expressed gene; FDR - false discovery rate; FPKM - fragments per kilobase of transcripts per million mapped fragments; GA - gibberellin; GA 3 - gibberellic acid 3; IAA - indole-3-acetic acid; KEGG - Kyoto encyclopedia of genes and genomes; $\log _{2} \mathrm{FC}-\log _{2}$ fold change; LR - lateral root; LRP - lateral root primordium; MR - main root; PEs - pectinesterases; PGs - polygalacturonases; PME - pectin methyl esterase; qPCR - quantitative PCR; TF - transcription factor.

Acknowledgements: We thank Prof. Robert Aiken of Northwest Research-Extension Center-Colby, Kansas State University for language improvement and valuable suggestions. The authors also thank Prof. A.H. Rajasab of Tumkur University for language improvement. This work was supported by the Institution National Natural Science Foundation of China, China, Project No. 31271652, and the Outstanding Youth Foundation of Heilongjiang Province, China, Project No. JC201309, the Training Project of Heilongjiang Bayi Agricultural University, China, Project No. XZR2015-12, the StartupFoundation for Doctors of Heilongjiang Bayi Agricultural University, Project No. XDB2014-14, XDB201817. The first two authors equally contributed to this work.

* Corresponding author; e-mail: zdffnj@263.net
} 
Gibberellins (GAs), a group of tetracyclic diterpenoid compounds, regulate diverse aspects of plant growth and development (Stamm et al. 2017, Manoharlal et al. 2018). Previous studies have found that GAs affect the growth and development of plant roots. In Arabidopsis, cell production rate and meristem size were unable to increase after germination in GA biosynthetic mutants ( $\mathrm{gal}-3$ and ga3ox1/ga3ox2), and exogenous gibberellins relieved this inhibition (Ubeda-Tomás et al. 2009). So, GAs regulate root growth by cell elongation and proliferation in Arabidopsis. Other studies suggest that GAs can promote MR growth. The MR length of gibberellin-deficient plants was shorter than that of wild type, which elongated after the application of GA. The dose-response experiments show that root growth is regulated by GA in a lower concentration range than is required for shoot growth (Tanimoto 2012). Gibberellins inhibitors delayed the growth of MR (Fu and Harberd 2003, Ueguchi-Tanaka et al. 2007). However, exogenous $\mathrm{GA}_{3}$ dramatically reduces the root growth in carrot, while the shoot growth of carrot is stimulated. It also significantly promotes xylem development in the tuberous root of carrot (Wang et al. 2015). In addition, the role of GAs in LR formation is also unclear (Fukaki and Tasaka 2009). Several lines of evidence suggest that GAs may inhibit LR formation. GAoverproducing mutants and GA application in aspen result in suppression of lateral and adventitious root formation (Eriksson et al. 2000). The LR proliferation and elongation are found in GA-insensitive (35S: PcGA2ox1) and GAdeficient (35S: rgl1) transgenic poplar (Gou et al. 2010). Overexpression of GAs biosynthesis gene in Arabidopsis (35S::AtGA20ox1) or the signal transduction gene in hybrid poplar (35S:PttGID1.1, 35S:PttGID1.3) resulted in the decrease of adventitious root number (Gou et al. 2010, Mauriat et al. 2014). So, the effects of exogenous GAs on root development vary depending on the plant species and experiment (Tanimoto 2012). Their roles in soybean root development and LR formation is poorly understood.

To reveal the regulation mechanism of GAs on root growth during seed germination, different content of GAs in root was established by seed soaking in gibberellic acid $\left(\mathrm{GA}_{3}\right)$ of different concentrations. We performed comprehensive analysis of seedling MR and LR morphological and anatomical characteristics, hormone content, and transcript profiles of genes to reveal the effects of GAs on root development. This study provided novel insights into the functions of GAs in soybean root growth during germination and early seedling growth.

\section{Materials and methods}

Soybean growth and $\mathbf{G A}_{3}$ application: The soybean [Glycine max (L.) Merr] cultivar Suinong28 was bred by Heilongjiang Academy of Agriculture Sciences, Heilongjiang Province, China. Gibberellic acid 3 (SigmaAldrich, St. Louis, MO, USA), was dissolved in absolute ethanol and then diluted with water to $1 \%(\mathrm{v} / \mathrm{v})$. The concentrations of $\mathrm{GA}_{3}$ used in this study were $0.43,0.87$, and $1.74 \mathrm{mM}$ corresponding to three different treatments named GA-15, GA-30, and GA-60. Air dry seeds (100) were soaked with $100 \mathrm{~cm}^{3}$ of $\mathrm{GA}_{3}$ solution $[0.1 \%$ ethanol $(\mathrm{v} / \mathrm{v})$ was used as a control] for $12 \mathrm{~h}$. Seeds were cultivated in sand as matrix moistened with the Hoagland nutrient solution in plastic containers for $13 \mathrm{~d}$ (16-h photoperiod with an irradiance of $300 \mu \mathrm{mol} \cdot \mathrm{m}^{-2} \cdot \mathrm{s}^{-1}$ and day/night temperatures of $25 / 18^{\circ} \mathrm{C}$ ). The experiments were carried out with three biological replicates with at least twenty plants in each replicate. We investigated the effect of exogenous $\mathrm{GA}_{3}$ on the soybean root for $13 \mathrm{~d}$ after $\mathrm{GA}_{3}$ treatment. Plant roots were harvested at 60,72 , and 84 $\mathrm{h}$ following the treatment to measure the number of LR primordia. Plant roots were harvested after 2, 3, 4, 5, 7, 9,11 , and $13 \mathrm{~d}$ for morphological observation. The MR and LR at $4 \mathrm{~d}$ were sampled for microscopic analyses. The MR at 1, 3, 5, and $10 \mathrm{~d}$ were used to measure the hormone content. Samples of MR treated with $0.87 \mathrm{mM} \mathrm{GA}_{3}$ and control at $3 \mathrm{~d}$ were frozen in liquid nitrogen immediately and used for the RNA-Seq and the real time qPCR.

Observation of root morphology and anatomy: To measure the effects of $\mathrm{GA}_{3}$ application on root morphology, the root samples were carefully removed from the sand, then washed with sterile water. In each experiment, twenty plants per treatment were chosen. Roots were scanned with an Epson Perfection V700 photo scanner (Seiko Epson Corporation, Nagano, Japan). The main morphological parameters of root system, including the length, number, diameter, surface area, and root volume, were determined using Image $J 1.63$ software. To observe the microscopic structure of the maturation zone of the roots, a $1-1.5 \mathrm{~cm}$ segments were excised from MR and LR tips. Samples were fixed in FAA solution containing $10 \mathrm{~cm}^{3}$ of $37 \%$ $(\mathrm{v} / \mathrm{v})$ formaldehyde, $5 \mathrm{~cm}^{3}$ of glacial acetic acid, $50 \mathrm{~cm}^{3}$ of $96 \%(\mathrm{v} / \mathrm{v})$ ethanol, and $35 \mathrm{~cm}^{3}$ of distilled water) for $24 \mathrm{~h}$, and then tissue slices were prepared for histological analysis according to $\mathrm{Li}$ (2009) and observed with an Olympus BX61 microscope (Olympus, Tokyo, Japan) and quantified using Image $J 1.63$ software.

Phytohormone analysis with HPLC-ESI-MS: Fresh mass $(1 \mathrm{~g})$ of the main roots was frozen in liquid nitrogen and then stored at a $-80{ }^{\circ} \mathrm{C}$ freezer until use. Each treatment has three biological replicates and five independent plants were collected in each replicate. The extraction, purification, and determinations of endogenous content of $\mathrm{GA}_{3}$, indole-3-acetic acid (IAA), and abscisic acid (ABA) were performed with an HPLC-ESI-MS technique as described by Gou et al. (2010). The experiment was carried out using a liquid chromatography-mass spectrometry system (LCQ Deca AMX, HPLC-ESI-MS; Thermo-Finnigan, San Jose, CA, USA) with C18 SepPak and MCX SPE columns (Qasis; Waters, Milford, MA, USA). The data were analyzed using the software Xcalibur 2.1 (Thermo-Finnigan) and quantified by reference to the internal standards using $\mathrm{M}^{+}$ratios in the equations for isotope dilution analysis.

Sequencing RNA: Ribonucleic acid was isolated from $200 \mathrm{mg}$ of soybean main roots collected at $3 \mathrm{~d}$ after treatment 
with solution bearing or lacking $\mathrm{GA}_{3}$. Three biological replicates were used for all RNA-seq experiments from each sample. Further RNA purification, cDNA synthesis and sequencing were performed as previously described (Han et al. 2017). Sequencing analysis was performed using a HiSeq 4000 platform (Illumina, San Diego, CA, USA) for paired end RNA sequencing (read length $2 \times$ $150 \mathrm{bp}$ ). The project and sequence data were submitted to the NCBI Bioproject PRJNA352344 and sequence read archive database under the accession number SRR5028739, SRR5028740, SRR5028741, SRR5028745, SRR5028746, and SRR5028747.

Sequenced RNA data analysis: Clean data without lowquantity reads (more than $50 \%$ of bases with a Q-value $\leq 10)$ were mapped to a reference genome $(G$. max V1.0) in the Phytozome database using TopHat (Kim et al. 2013). Subsequently, the expression of each transcript was calculated and normalized to obtain fragments per kilobase of transcript per million fragments mapped (FPKM). Then, $P$-values and $\log _{2}$ fold change $\left(\log _{2} \mathrm{FC}\right)$ were calculated. Only the gene with false discovery rate (FDR) $\leq 0.05$ and $\mid \log _{2}$ FC $\mid \geq 1$ were considered as significant differentially expressed genes (DEGs) between the $\mathrm{GA}_{3}$ treatment and control samples. For function analysis, DEGs were aligned to $N C B I$ non-redundant protein $(N r)$, Swiss-Prot, gene ontology (GO, http://www.geneontology.org/), clusters of orthologous groups $(C O G)$, and the Kyoto encyclopedia of genes and genomes $(K E G G)$ databases. To predict transcriptional networks in soybean roots after $\mathrm{GA}_{3}$ treatment, Arabidopsis transcription factors (TFs) that were most similar to the soybean TFs and also in the Arabidopsis transcriptional regulatory map dataset (ATRM, http://atrm.cbi.pku.edu.cn/) were selected to generate the transcription networks. Cytoscape v. 3.2.1 was used to visualize of the transcription networks (Smoot et al. 2011).

Real time quantitative PCR analysis: 34 genes involved in GA, auxin, cytokinin, abcisic acid and brassinolide pathways, TFs, and genes related to cell elongation and cell wall modification were selected from G. max database in joint genome institute (http://jgi.doe.gov/). The PCR primer pairs of all genes are shown in Table 1 Suppl. The actin (Glyma18 g52780.1) and eukaryotic elongation factor 1 ( $L F 1 B$, Glyma02g44460.1) gene of soybean was selected as an internal control.

The MR samples of GA-30 and control at 1, 3, and $10 \mathrm{~d}$ were used for real time qPCR. The extraction and detection of total RNA were carried out as in RNA-seq experiment. The cDNA was made with RNA LA PCR kit (AMVver 1.1; (Takara, Dalian, China) and qPCR was performed using Takara SYBR Premix Ex Taq in a total of volume of $20 \mathrm{~mm}^{3}$
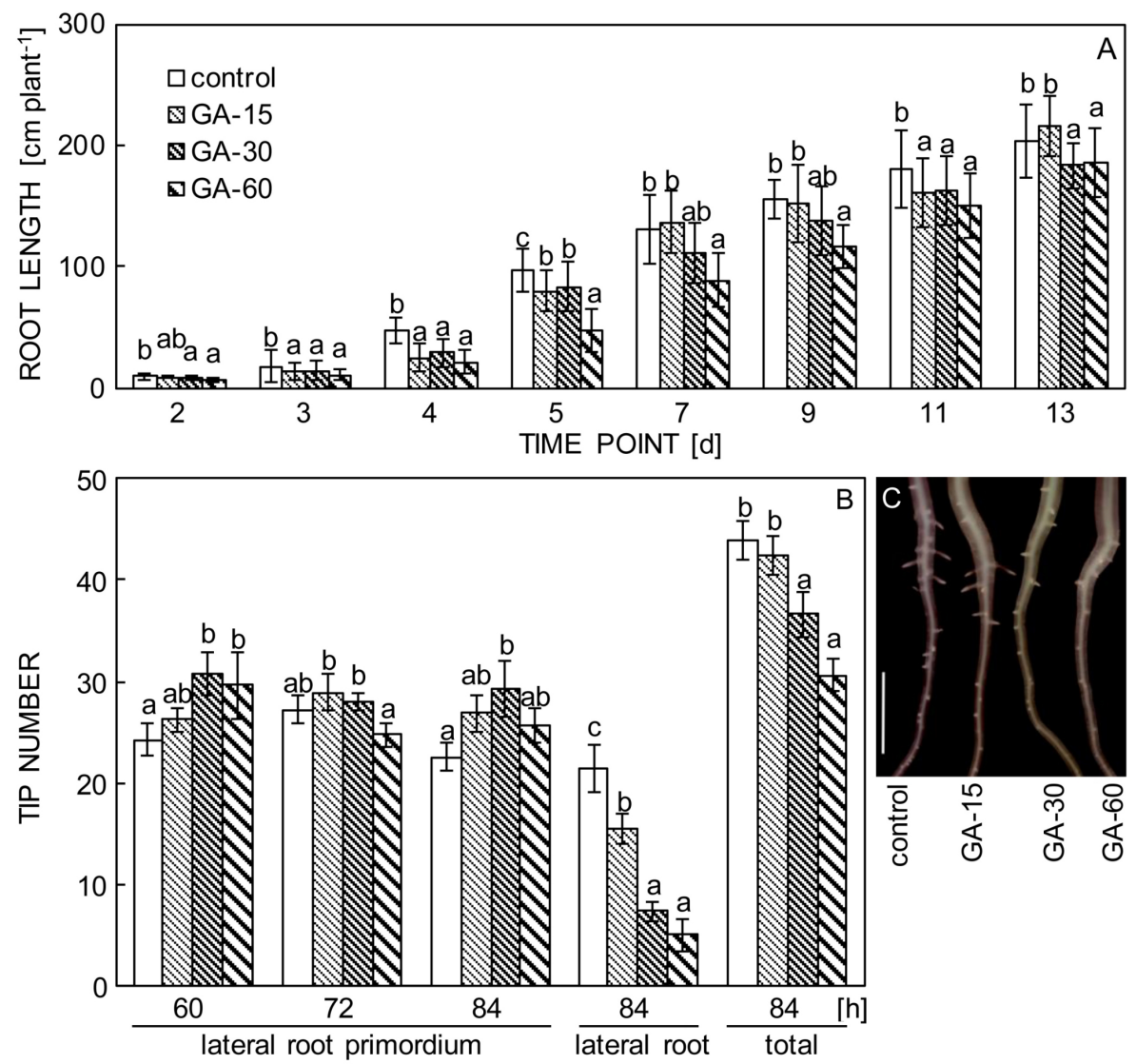

Fig. 1. Effect of gibberellic acid $3\left(\mathrm{GA}_{3}\right)$ treatment on root length $(A)$ and the number of root tips $(B)$. Soybean seeds soaked in $0.43,0.87$, and $1.74 \mathrm{mM} \mathrm{GA}_{3}$ corresponding to three different treatments GA-15, GA-30, and GA-60. Means \pm SE, $n \geq 20$, different letters show means which differ significantly $(P \leq 0.05)$ according to one way ANOVA combined with the Tukey's multiple comparison posttest. Photographs of representative roots $3 \mathrm{~d}$ after treatments with different concentration of $\mathrm{GA}_{3}(C)$. The bar represents $1 \mathrm{~cm}$. 
containing $10 \mathrm{~mm}^{3}$ of SYBR Premix Ex Taq, $0.4 \mathrm{~mm}^{3}$ of $R O X$ reference dye, $7.0 \mathrm{~mm}^{3}$ of deionized water, $0.8 \mathrm{~mm}^{3}$ of each forward and reverse primer, and $1 \mathrm{~mm}^{3}$ of diluted cDNA strand $(100 \times)$. The qPCR analysis was performed in an ABI 7500 thermocycler (Applied Biosystems, Foster City, CA, USA). PCR cycling was performed at $95{ }^{\circ} \mathrm{C}$ for $30 \mathrm{~s}$, followed by 40 cycles of $95^{\circ} \mathrm{C}$ for $5 \mathrm{~s}, 60^{\circ} \mathrm{C}$ for $30 \mathrm{~s}$. Data were transformed and analyzed by the $2^{-\Delta \Delta \mathrm{Ct}}$ method (Rao et al. 2013), and relative expressions of each gene in soybean roots were analyzed using the Applied Biosystems 7500 SDS V 2.0 qPCR software.

Statistical analysis. One way $A N O V A$ with LSD or the
Tukey's multiple comparison posttest was applied to detect differences under different treatments at the 0.05 significance level.

\section{Results}

Soybean seeds were treated with three different concentrations of $\mathrm{GA}_{3}$, and surveyed for $13 \mathrm{~d}$. The root length and root surface area decreased under $\mathrm{GA}_{3}$ treatments at multiple time periods; GA-60 treatment had the greatest impact (Fig. 1A, Fig. 1 Suppl.). All GA 3 treatments significantly reduced the root volume (Fig. 1
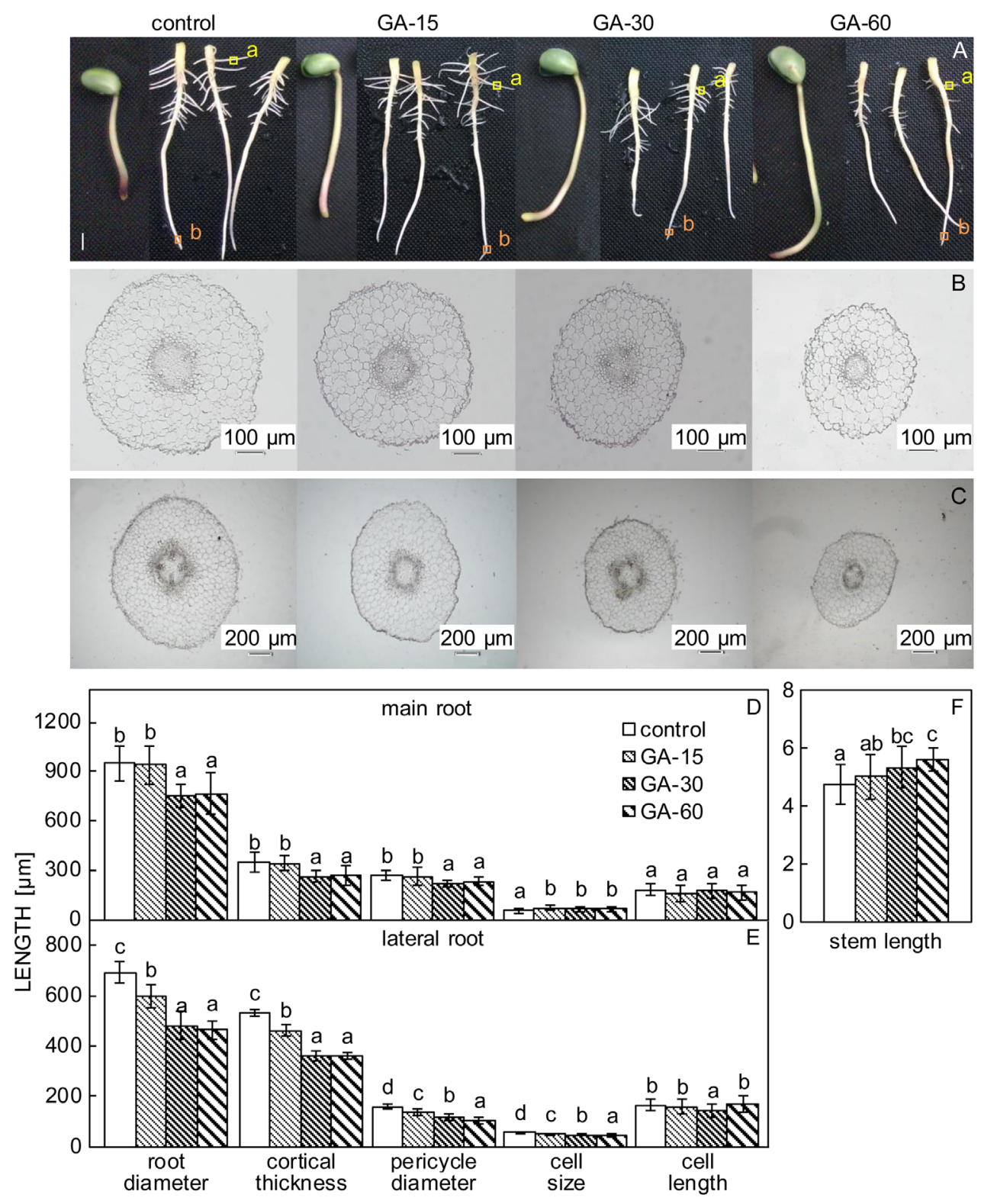

Fig 2. The change of morphology and anatomy of shoots and roots $96 \mathrm{~h}$ after gibberellin treatments. $A$ - The morphology of shoot and root; the bar represents $1 \mathrm{~cm}$; a - sample location in a lateral root, $\mathrm{b}$ - sample location in the main root. $B$ - The microscopic structures of a segment excised $1-1.5 \mathrm{~cm}$ from a lateral root tip; the $b a r$ represents $100 \mu \mathrm{m}$. $C$ - The microscopic structures of segment excised $1-1.5 \mathrm{~cm}$ from the main root tip; the bar represents $200 \mu \mathrm{m} . D$ - Quantification of different anatomical parameters in the main and lateral roots. Means \pm SEs, $n=3$, different letters show means which differ significantly $(P \leq 0.05)$. 
Suppl.). The diameter of MR decreased gradually with the increase of $\mathrm{GA}_{3}$ concentration at 3,4 , and $5 \mathrm{~d}$, and the diameter of $\mathrm{MR}$ in all treatment groups remained lower than that of control at 9 and $11 \mathrm{~d}$. The change of LR diameter was similar with that of MR diameter, and the diameter of LR in all treatment groups was lower than that of control at $3,4,7$, and $9 \mathrm{~d}$. With the further growing of plant, the effect of $\mathrm{GA}_{3}$ on soybean root diameter diminished; there was no significant effect after $11 \mathrm{~d}$ (Fig. 1 Suppl.).

Tip number, a measure of LR formation, of treated plants was significantly less than that of the control group at $4 \mathrm{~d}$ (Fig. 1 Suppl.). The result indicated the LR formation decreased. However, the quantity of lateral root primordia (LRP) of soybean increased after $\mathrm{GA}_{3}$ treatment. The number of LRP increased in GA-30 and GA-60 treatments at $60 \mathrm{~h}$ and GA-30 treatment group at $84 \mathrm{~h}$ (Fig. $1 B$ ). The quantity of LRP also significantly increased at GA-30 treatment after $84 \mathrm{~h}$. However, many LRP of treated plants did not form LR, as indicated by fewer LR in treated plants as compared with control at $84 \mathrm{~h}$. With increased $\mathrm{GA}_{3}$ concentration, the number of LR decreased. The number of LR in GA-30 and GA-60 treated plants were significantly less than that of control (Fig. $1 B, C$ ). Thus, $\mathrm{GA}_{3}$ treatments affected the MR and LR development.

Epidermis, cortex, and vascular cylinder comprise the soybean root system, jointly determining the morphology of plant root. $\mathrm{GA}_{3}$ treatment changed the morphology of soybean root at $4 \mathrm{~d}$. With the increase of $\mathrm{GA}_{3}$ concentration, the length of hypocotyl increased (Fig. $2 A$ ), while the diameter of LR and MR decreased (Fig. 2D,E). The microscopic observations of transverse sections of LR and MR showed that the decrease in diameter resulted from reduced cortical thickness and pericycle diameter. The size of cortical cell also changed (Fig. 2B,C). However, $\mathrm{GA}_{3}$ treatment had no obvious effect on root cell length (Fig. 2D).

We assayed the amount of $\mathrm{GA}_{3}$, IAA, and ABA in root at $1,3,5$, and $10 \mathrm{~d}$, after $\mathrm{GA}_{3}$ treatments respectively. As shown in Fig. $3 A$, the $\mathrm{GA}_{3}$ content of treated plant roots was greater than that of the control group. The $\mathrm{GA}_{3}$ content in roots of treatment group and control group gradually decreased over time. $\mathrm{GA}_{3}$ treatment also affected the content of IAA in root to some extent (Fig. $3 B$ ). At $1 \mathrm{~d}$, the content of IAA in GA-30 and GA-60 groups significantly decreased, and the content of IAA in GA-15 group at $3 \mathrm{~d}$, in GA-60 group at $5 \mathrm{~d}$, and in GA-15 group at $10 \mathrm{~d}$ significantly increased. As concerns $\mathrm{ABA}, \mathrm{GA}_{3}$ application increased the content of $\mathrm{ABA}$ in treatment groups at 1, 3, and $5 \mathrm{~d}$ but at $10 \mathrm{~d}$, the content of ABA in GA-60 group decreased (Fig. 3C).

In MR, changes in transcriptome determined $3 \mathrm{~d}$ after treatment with solution bearing or lacking $\mathrm{GA}_{3}$ indicated that reads mapping to the genome sequence made up approximately $85 \%$ of the reads (Table 2 Suppl.). A total of 1317 DEGs were identified between $\mathrm{GA}_{3}$-treated (GA-30) and untreated (control) samples by FPKM, and 940 DEGs were down-regulated, while 377 DEGs were activated (Fig. 2 Suppl.). The number of downregulated

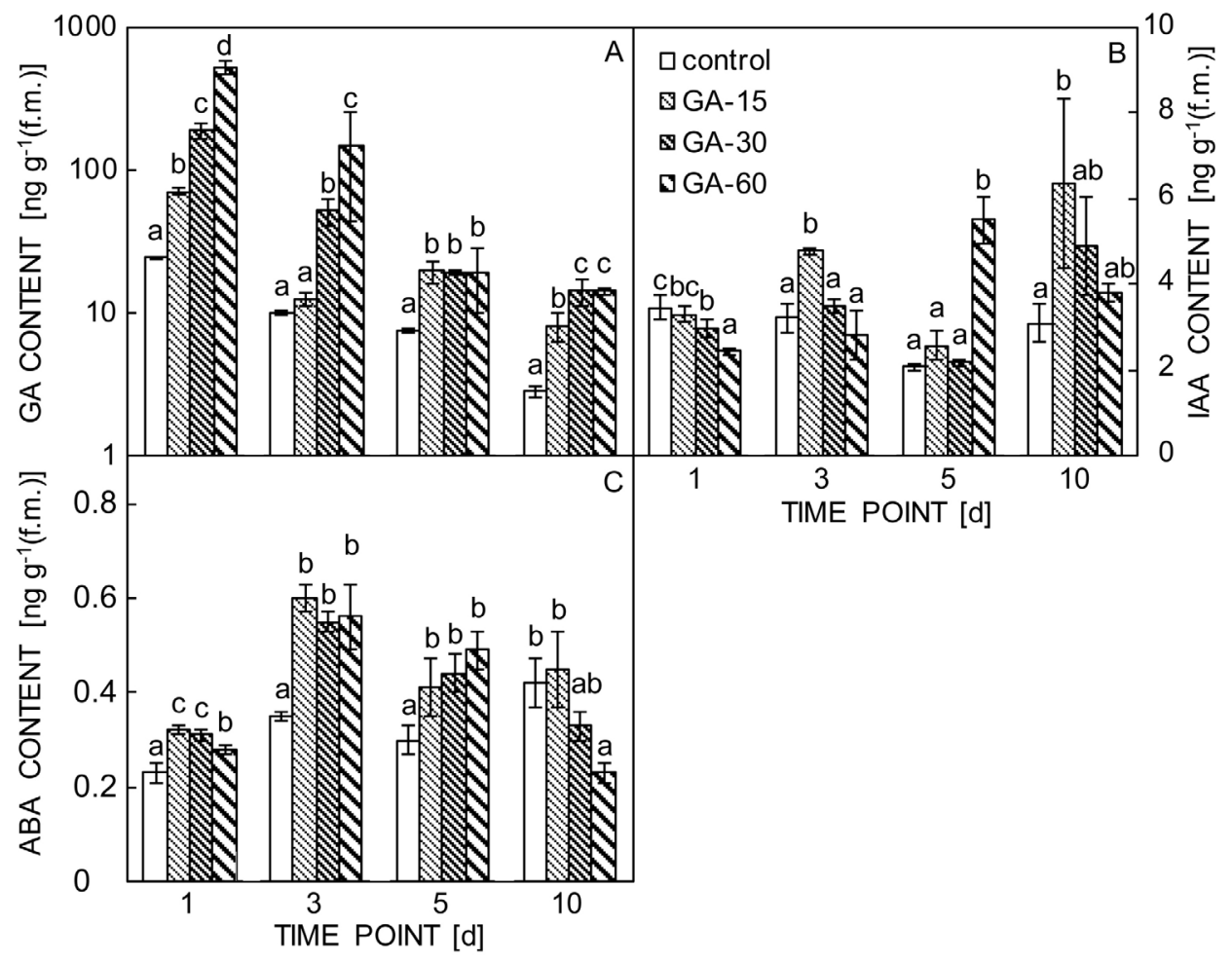

Fig. 3. Content of phytohormones in soybean roots in response to gibberellic acid $3\left(\mathrm{GA}_{3}\right)$ treatment. The phytohormones content was measured at $24,72,120$, and $240 \mathrm{~h}$ after treatments with different concentrations of $\mathrm{GA}_{3}$. $A$ - gibberellins (GA) content, $B$ - abscisic acid (ABA) content; $C$ - indole-3-acetic acid (IAA) content. Means \pm SEs, $n=3$, different letters show means which differ significantly $(P \leq 0.05)$. 
Table 1. Effects of gibberellic acid $3\left(\mathrm{GA}_{3}\right)$ on the expressions of genes relating to gibberellin biosyntheses and signaling pathways.

\begin{tabular}{|c|c|c|}
\hline Gene ID (Wm82.a2.v1) & Swissprot_annotation & $\log _{2} \mathrm{FC}$ \\
\hline Glyma.03G131200 & gibberellin 20 oxidase 2 & -1.02 \\
\hline Glyma.04G070500 & gibberellin 20 oxidase 1 & 1.31 \\
\hline Glyma.19G062800 & gibberellin 20 oxidase 1 & -1.19 \\
\hline Glyma.19G062600 & gibberellin 20 oxidase 1 & -1.48 \\
\hline Glyma.18G061300 & gibberellin 2-beta-dioxygenase 8 & -3.61 \\
\hline Glyma.13G259500 & gibberellin 2-beta-dioxygenase 1 & 1.93 \\
\hline Glyma.02G185200 & AP2-like ethylene-responsive transcription factor At2g41710 & -1.39 \\
\hline Glyma.02G239400 & probable acyl-activating enzyme 5 , peroxisomal & 4.37 \\
\hline Glyma.13G189800 & gibberellin receptor GID1C & 2.04 \\
\hline Glyma.15G049000 & G-box-binding factor 4 & 1.90 \\
\hline Glyma.16G063700 & Myb-related protein 315 & 1.85 \\
\hline Glyma.02G067600 & ethylene-responsive transcription factor LEP & 1.20 \\
\hline Glyma.U038900 & transcription factor RAX3 & 1.04 \\
\hline Glyma.17G258200 & Snakin-2 (Precursor) & 1.13 \\
\hline Glyma.14G219100 & Snakin-2 (Precursor) & 1.41 \\
\hline Glyma.12G238700 & transcription factor bHLH130 & -1.03 \\
\hline Glyma.04G101900 & transcription factor MYB39 & -2.07 \\
\hline Glyma.06G103300 & transcription factor MYB39-like & -3.60 \\
\hline Glyma.11G194100 & transcription factor RAX2 & -1.00 \\
\hline Glyma.06G043400 & F-box protein GID2 & -1.59 \\
\hline Glyma.13G152900 & probable acyl-activating enzyme 12 , peroxisomal & -1.88 \\
\hline Glyma.18G044900 & probable acyl-activating enzyme 12 , peroxisomal & -1.06 \\
\hline Glyma.13G200400 & flavin-containing monooxygenase FMO GS-OX5 & -1.08 \\
\hline Glyma.13G113100 & probable flavin-containing monooxygenase 1-like & -4.12 \\
\hline Glyma.08G021900 & probable WRKY transcription factor 53 & -1.29 \\
\hline Glyma.09G212500 & growth-regulating factor 4 & -1.03 \\
\hline
\end{tabular}

genes was 2-times higher than that of upregulation ones. Using $\left|\log _{2} \mathrm{FC}\right| \geq 3$ as a cutoff value, 155 genes in the root showed differential expression patterns between the treatment and the control (Table 3 Suppl.). There were 27 genes with increased transcript abundance and 128 genes with decreased transcript abundances, and the number of downregulated genes were 4.7-times higher than that of upregulated genes. Function analysis of DEGs were conducted in various databases (Fig. 3 Suppl. and Fig. 4 Suppl.).

A large number of DEGs involving in GA biosynthetic and signalling pathways were identified (Table 1). For GA biosynthesis pathway, most of GA20ox 2 genes (Glyma.03G131200) and GA20ox 1 genes (Glyma.19G062800 and Glyma.19G062600) were downregulated, while Glyma.04G070500 (GA20ox 1) was up-regulated in root after $\mathrm{GA}_{3}$ application. The genes of GA2ox which encode the GA-inactivating enzymes exhibited differential expression after the application of $\mathrm{GA}_{3}$, Glyma.18G061300 (GA2ox 1) was up-regulated by $\log _{2}$ FC of 1.93 . These changes coincided with the feedback mechanism of the responsiveness of tissue to the bioactive GAs influencing GA metabolism (Cheng et al. 2015). Glyma.13G259500 (GA2ox 8) which hydroxylates the C20-GA GA 12 and $\mathrm{GA}_{53}$ (Schomburg et al. 2003), was down-regulated by $\log _{2} \mathrm{FC}-3.61$.
This feedback mechanism also appeared to play a role at the GA perception. For GA signalling pathway, $\mathrm{GA}_{3}$ negatively regulates the expression of gene GID2 encoding the gibberellin receptor F-box protein, of gene GS-OX5 encoding acyl-activating enzyme 12, flavin-containing monooxygenase (FMO), and of some transcription factors, such as bHLH130, MYB39, MYB39-like, RAX2, $W R K Y 53$, etc. In contrast, the transcription of probable acyl-activating enzyme 5, gibberellin receptor GID1C, G-box-binding factor 4, Myb15, LEP, RAX3, and Snakin-2 exhibited up-regulation following $\mathrm{GA}_{3}$ treatment (53\% genes belonged TFs among those GA signalling pathwayrelated genes). The RNA-seq results revealed that $\mathrm{GA}_{3}$ not only obviously changed expression pattern of the GA biosynthesis genes, but also affected some regulation genes of GA-related pathway.

The TFs play important roles in regulation of gene expression of all kinds of processes including plant development. In DEGs, there are 103 TFs, accounting for $7.8 \%$ of total DEGs. Those TFs belonged to 15 transcription factor families (Fig 4A) classified. The most highly represented TF families were the WRKY (which is a class of DNA-binding proteins that recognize the TTGAC(C/T) W-box elements), bHLH (basichelix-loophelix), ERF, MYB (myeloblastosis), and NAC families.

We constructed the transcriptional regulation network 
of these TFs in soybean roots affected by $\mathrm{GA}_{3}$ treatment according to ATRM. As shown in Fig. $4 B$, there are 9 $\mathrm{TF}$ interaction nets among $103 \mathrm{TFs}$, and only one net has different nodes, the others are simple. The complex transcriptional regulation net included WRKY6, WRKY33, and $W R K Y 70$.

Further, $\mid \log _{2}$ FC $\mid \geq 3$ TFs were focused on. There are 2 TFs up-regulated and 9 TFs down-regulated after $\mathrm{GA}_{3}$ treatment. $B E E 3$ (brassinosteroid enhanced expression 3) -like (Glyma.09G183500) showed $\log _{2}$ FC 3.76, which was annotated in GA mediated signalling pathway (GO:0009740), and biological process root hair cell development, and has the positive regulation of brassinosteroid biosynthesis (GO:2000488) according to $G O$ database. BR-induced $\mathrm{BEE} 3$ is required for a proper BR response, and plays a role in stem growth by increasing the proliferation of xylem cells to promote the initial thickening growth of stems in poplar (Noh et al. 2015). BEE3 is also regulated by other hormones, notably ABA (Friedrichsen et al. 2002). In this study, we also confirmed BEE3-like TFs response to $\mathrm{GA}_{3}$ treatment in root. Among these down-regulated TFs, WRKY51 and WRKY70 both participated in lateral root development (GO: 0048527). Meanwhile, these down-regulated TFs were all in the hormone-mediated signalling pathway. WRKY40 responded to ABA, jasmonic acid (JA), and SA. WRKY51 responded to ethylene and auxin, and it was included in the JA mediated signalling pathway. WRKY67 responded to JA and it was included in the SA mediated signalling pathway. WRKY70 played a role in the SA and JA mediated signalling pathways. So, the exogenous $\mathrm{GA}_{3}$ application affected the expression of genes related to different hormones at the same time.

$K E G G$ analysis of DEGs showed that many genes in multiple metabolic pathways responded to $\mathrm{GA}_{3}$ treatment. These include plant hormone signal transduction, phenylpropanoid biosynthesis, and plant-pathogen interaction (Fig. 5, Table 4 Suppl.).

Exogenous $\mathrm{GA}_{3}$ affected the transduction pathways of hormones other than GA. As concerns auxins, exogenous

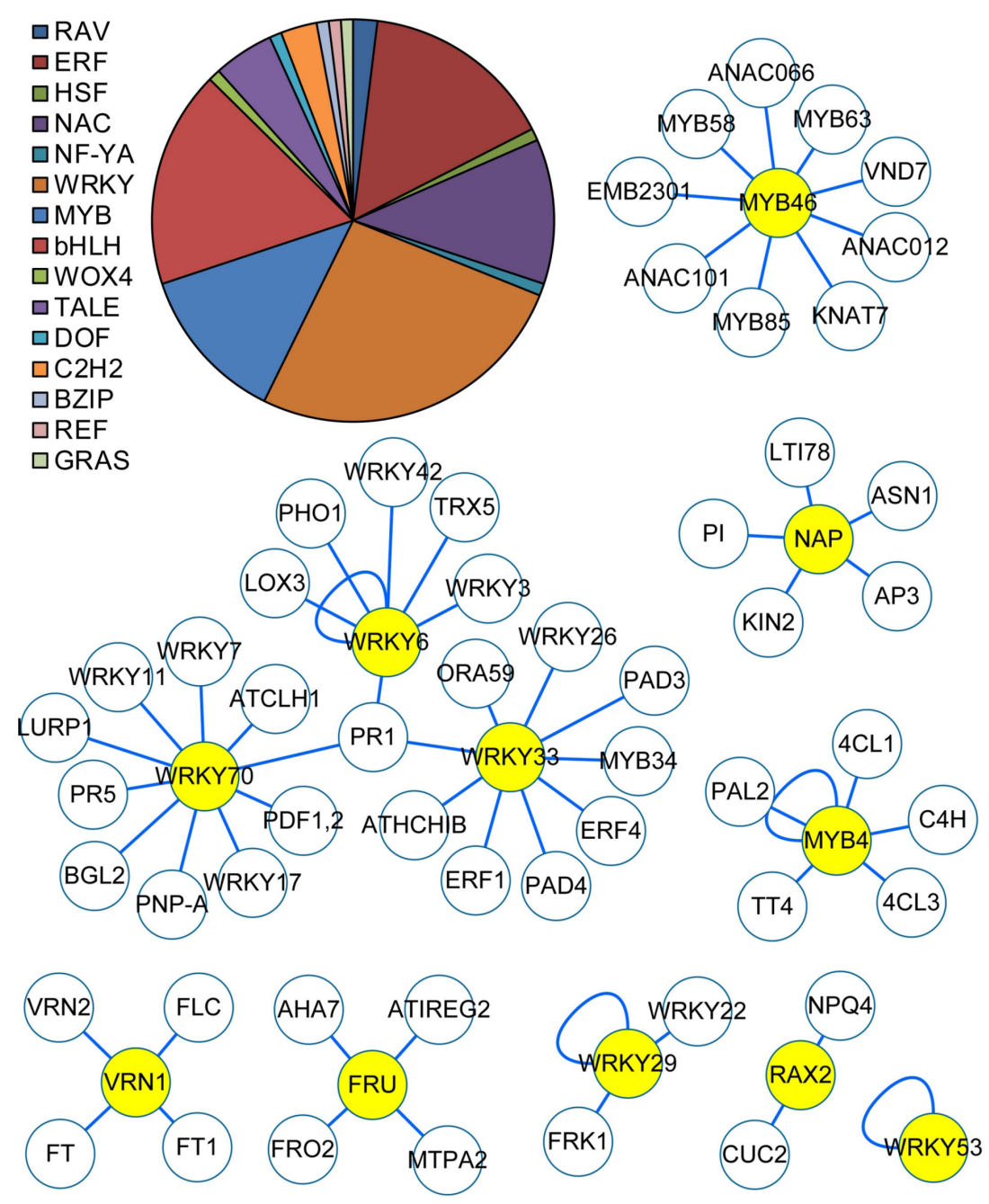

Fig. 4. The transcriptional network of differentially expressed transcription factors (TFs) in soybean roots after gibberellic acid $3\left(\mathrm{GA}_{3}\right)$ treatment. The Arabidopsis TF orthologs identified in the expression data of soybean roots after $\mathrm{GA}_{3}$ treatments were color-coded. Visualization of the network was generated by Cytoscape v. 3.2.1. Arrows indicate a positive regulation of the target TFs or co-regulators generated from the ATRM data set. 
$\mathrm{GA}_{3}$ increased the transcription of auxin responsive $G H 3$ gene family and decreased the transcription of small auxin-up RNA (SAUR). GH3 and SAUR were auxinresponsive genes which were reported to play a role in cell enlargement and plant growth (Takase et al. 2004; Farquharson 2014). As concerns cytokinins, histidinecontaining phosphotransfer protein genes (HPts) were down-regulated which were annotated as AHP4. AHP4 in

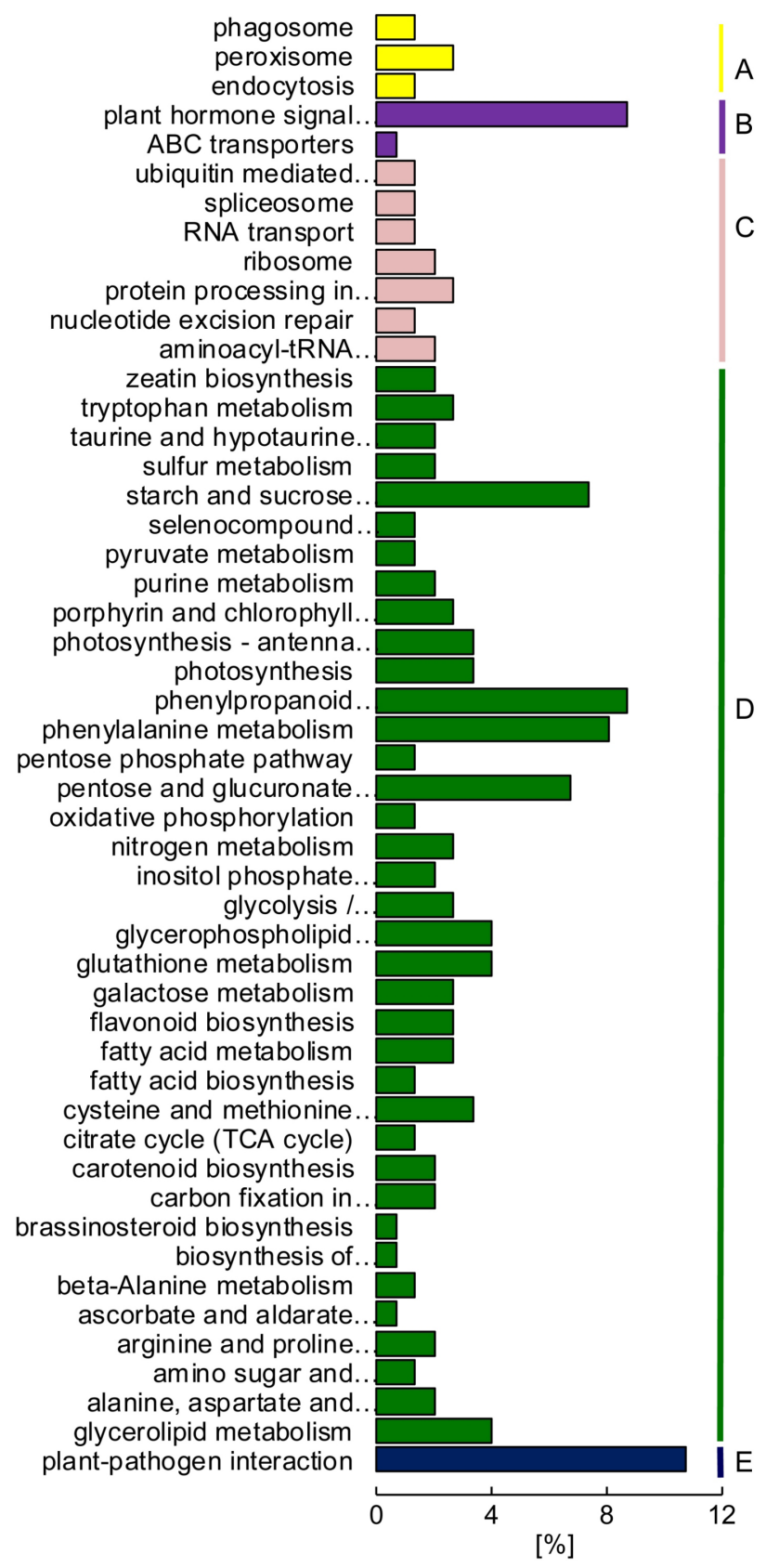

Fig. 5. The Kyoto encyclopedia of genes and genomes (KEGG) metabolism pathway categories of assembled unigenes: cellular processes $(A)$, environmental information processing $(B)$, genetic information processing $(C)$; the main functional categories are metabolism $(D)$ and organismal systems $(E)$. Bars represent the numbers of Glycine $\max$ assignments of unigenes with BLASTX matches to each $K E G G$ term.
Arabidopsis is a mediator in a multistep phosphorylation pathway for cytokinin signalling and negatively regulated thickening of the secondary cell wall of the anther endothecium (Jung et al. 2008). As concerns ABA, GA 3 application increased transcriptions of $\mathrm{ABA}$ responsive element binding factor $(E R F 1 / 2)$. The transcript of ethyleneresponsive transcription factor 1 in ethylene signalling pathway decreased after $\mathrm{GA}_{3}$ treatment. Probable WRKY transcription factor 40 (Glyma.17G222300, log FC -5.29), a negative regulator of ABA-dependent inhibition of seed germination, relocalized from PNBs to the nucleoplasm in the presence of ABA in a dynamic and phosphorylationdependent manner (Katja and Maik 2015). Brassinosteroid insensitive 1-associated receptor kinase $1 B A K 1$ and BRI1 kinase inhibitor $1 B K I 1$, which acted in brassinosteroid signal pathway, showed decreased expression. The transcript of jasmonate ZIM domain-containing protein JAZ was up-regulated (Fig. 5 Suppl., Table 5 Suppl.) which played versatile roles in multiple aspects of plant development and defense (Yuan et al. 2017).

The $\mathrm{GA}_{3}$ treatment also affect the transcription of some genes in the phenylpropanoid metabolic pathway which is related to the synthesis of many secondary metabolites, such as lignin and flavonoids. This pathway affects plant growth and development together with their adaptation to the environment. DEGs involved in phenylpropanoid pathway were identified in this study (Fig. 6 Suppl.). The transcription of trans-cinnamate 4-monooxygenase (Glyma.20G114200) was downregulated, and the transcription of erulate-5-hydroxylase genes (Glyma.16G131200) were up-regulated. The transcription gene concerning peroxidases were also changed, 13 genes up-regulated and 10 genes downregulated. Flavonoid pathway is a branch pathway in the common phenylpropanoid pathway. Many genes in this pathway were markedly decreased, including genes of trans-cinnamate-4-monooxygenase (Glyma.20G114200), chalcone isomerase (Glyma.20G241700), flavonoid3'-monooxygenase (Glyma.05G021800), flavanone4-reductase (Glyma.18G220500, Glyma.09G269500). Many genes in signal pathway of plant-pathogen interaction were identified. In soybean root, transcriptions of most genes were down-regulated by exogenous $\mathrm{GA}_{3}$ treatment, such as genes of mitogen-activated protein kinase kinase $1(M A P 2 K 1)$ in MAPK signalling pathway, transcription factor WRKY29, WRKY33, calcium-binding protein $C M L$, and respiratory burst oxidase.

GA-dependent expression of cell wall-related enzymes occurred in several species during seed germination (Ren and Kermode 2000, Chen et al. 2001, Ogawa et al. 2003). Among GA regulated genes identified in this study, there were some classes of genes implicated in the biogenesis and degradation of cell wall. As shown in Table 6 Suppl., 55 DEGs were classified as genes putatively involved in changing cell-wall composition and properties, which may affect cell-wall development in soybean root. Among cell-wall-related DEGs, 44 genes were down-regulated, including 12 glucosidases, 10 pectinesterases $(P E S), 5$ polygalacturonases $(P G s), 8$ extension (EXT), 1 expansins (EXPS), $2 \beta$-1,3-glucanase, 
1 glycogenin glucosyltransferase, 2 pectatelyases $(P L s)$, $1 \beta$-galactosidases, 1 glucan endo-1,3- $\beta$-glucanase, 1 acid $\beta$-fructofuranosidase, 1 cellulose synthase, and 1 chitinase. After $\mathrm{GA}_{3}$ treatment, some genes were up-regulated, such as $6 P G s, 1$ glucan endo-1,3- $\beta$-glucosidase 14, 1 endoglucanase, $2 \mathrm{PES}$, and 1 cellulose synthase. Extensins, hydroxyproline-rich glycoproteins (HRGPnt3), a class of structural proteins of cell wall, play roles in cell wall assembly, cell shape and size, and disease resistance (Fan et al. 2017). Treatment with $\mathrm{GA}_{3}$ down-regulated expression of $8 E X T s$ genes.

In order to verify the reliability of transcriptome data, we selected 36 DEGs for RT-qPCR, which were genes in the hormones related pathways or which we were interested. The quantitative expression analysis of 36 genes were accomplished (Table 7 Suppl.). We detected the expression of $3 E X T s$, which were the cell wall related genes. The results showed that 3 EXTs all downregulated at $1 \mathrm{~d}$, and expression still kept in low level at $3 \mathrm{~d}$. Until $10 \mathrm{~d}$, its expression recovered (Fig. 6). Two GA biosynthesis genes GA20OX1-1 and GA20OX1-2 were down-regulated after $\mathrm{GA}_{3}$ treatment at the early stage, and the expression recovered or ascended at $10 \mathrm{~d}$ to some extent. The expression profile of GA20OX1 was most correlated with changes in $\mathrm{GA}_{3}$ increase at the early stage. TF VNR1 was down-regulated, and kept in a low expression levels. Expression level of $A S$ (hydroquinone glucosyltransferase) in sugar metabolism was significantly up-regulated, and recovered till 10 d. GH3.1 (indole-3acetic acid-amido synthetase) was obviously up-regulated, and downed to the normal level at $10 \mathrm{~d}$, which belongs to the auxin responsive $G H 3$ gene family.

The correlation analysis by Pearson method showed that transcriptome data was positively correlated with the quantitative data, and correlation coefficient was 0.853 (Table 8 Suppl.). These results indicated that the transcriptome data was reliable.

\section{Discussion}

The application of plant growth regulators is an important way to improve crop traits. GAs have been widely used to regulate seed germination, plant growth, and fruit yield (Peng 2002). Root system is one of the main factors in maintaining crop yield and quality, and the LR is a vigorous and physiologically active part of the soybean root system. Therefore, the effects of $\mathrm{GA}_{3}$ on soybean root growth, especially LR growth provides opportunity to gain insight to soybean root system development. In this paper, we performed a detailed analysis of the effect of $\mathrm{GA}_{3}$ treatment on root development from different aspects: morphological characteristics of roots, root microstructure, hormonal changes, and genes expression profile.

Previous studies have focused on the effects of mutations of GAs synthetic gene on roots. Inhibition

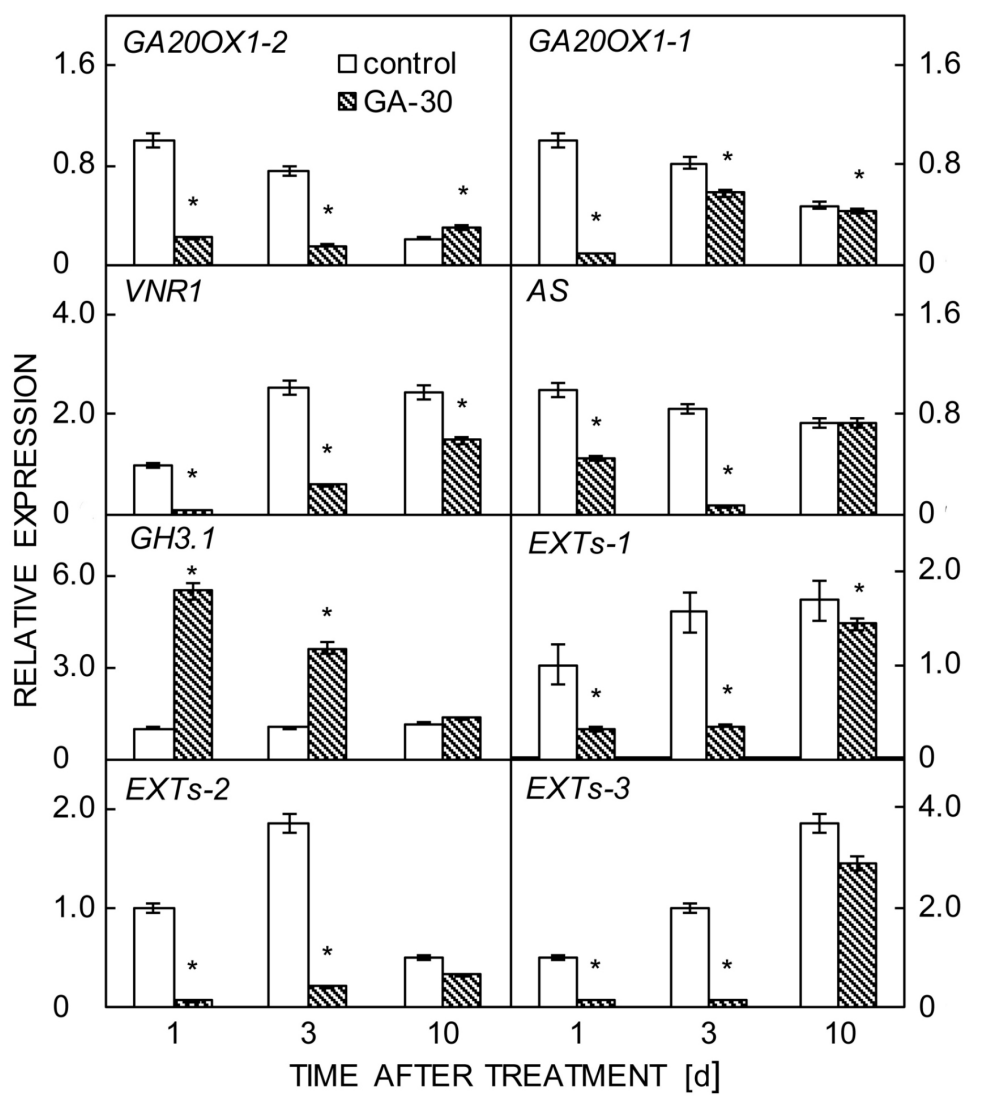

Fig. 6. The reverse transcription quantitative PCR analysis of eight differentially expressed genes (GA20OX1-1, GA20OX1-2, VNR1, $\left.A S, G H 3.1, E X T_{s}-1, E X T_{s}-2, E X T_{s}-3\right)$ in soybean roots treated with gibberellic acid 3. 
effect on root development caused by the mutation of GAs biosynthesis gene showed that GAs have a positive effect on root system (Fu and Harberd 2003, Achard et al. 2009, Ubeda-Tomás et al. 2009). We investigated the role of GAs by seed soaking with $\mathrm{GA}_{3}$. Excessive $\mathrm{GA}_{3}$ did not show positive effect on root, on the contrary it inhibited the breaking of LRP through cortex to form the LR (Fig. 1). Meanwhile, the root diameter, surface area, and volume of root significantly decreased in the early stages. In poplar and carrot, the application of $\mathrm{GA}_{3}$ also had the negative effect on root growth (Gou et al. 2010, Wang et al. 2015). Tanimoto (2012) reported GA-suppressed thickening of roots usually observed in the elongation zone of the root and mainly caused by the expansion of cortical cells. Our microscopic examination of anatomical structures showed that $\mathrm{GA}_{3}$ treatment changed the thickness of the cortex, the diameter of the pericycle, and the cell size (Fig. 2). In our previous study, uniconazole treatment promoted root growth in soybean which indirectly indicated the inhibition effect of $\mathrm{GA}_{3}$ on root growth (Han et al. 2017). The effects of exogenous GAs and GAs inhibitors on root development vary depending on the plant species and experimental conditions (Tanimoto 2012), so more studies in other species will provide further information.

$\mathrm{GA}_{3}$ treatment altered morphological parameters, anatomical structure, and transcriptional regulatory networks in soybean root. $\mathrm{GA}_{3}$ treatment restrained root growth that maybe related to the transverse growth of cell. Morphological parameters also showed that GA treatment inhibited the penetration of the LR primordia through the cortex. The $\mathrm{GA}_{3}$, IAA, and $\mathrm{ABA}$ content in soybean root changed after $\mathrm{GA}_{3}$ application. RNA-seq results suggested that many GA biosynthesis and signalling pathway genes, other hormones signalling pathway genes, TF genes, and cell wall related proteins, especially cell-wall-loosening proteins significantly changed their expression profile, which may be related to the morphogenesis change, the inhibition of LR, and the change of hormone content.

\section{References}

Achard, P., Gusti, A., Cheminant, S., Alioua, M., Dhondt, S., Coppens, F., Beemster, G.T.S., Genschik, P.: Gibberellin signaling controls cell proliferation rate in Arabidopsis. - Curr Biol. 19: 1188, 2009.

Chen, F., Dahal, P., Bradford, K.J.: Two tomato expansin genes show divergent expression and localization in embryos during seed development and germination. - Plant Physiol. 127: 928936, 2001.

Cheng, C., Jiao, C., Singer, S.D., Gao, M., Xu, X., Zhou, Y., Li, Z., Fei, Z., Wang, Y., Wang, X.: Gibberellin-induced changes in the transcriptome of grapevine (Vitis labrusca $\times$ V. vinifera) cv. Kyoho flowers. - BMC Genomics 16: 128, 2015.

Eriksson, M. E., Israelsson, M., Olsson, O., Moritz T.: Increased gibberellin biosynthesis in transgenic trees promotes growth, biomass production and xylem fiber length. - Nat Biotechnol. 18: 784-788, 2000.

Fan, C., Li, Y., Hu, Z., Hu, H., Wang, G., Li, A., Wang, Y., Tu, Y., Xia, T., Peng, L.: Ectopic expression of a novel OsExtensinlike gene consistently enhances plant lodging resistance by regulating cell elongation and cell wall thickening in rice. Plant Biotechnol. J. 16: 254-263, 2017.

Farquharson, K.L.: SAUR19 links auxin and plasma membrane H+-ATPases in cell expansion. - Plant Cell 26: 1835, 2014.

Friedrichsen, D.M., Nemhauser, J., Muramitsu, T., Maloof, J N., Alonso, J., Ecker, J.R., Furuya, M., Chory, J.: Three redundant brassinosteroid early response genes encode putative bHLH transcription factors required for normal growth. - Genetics 162: 1445, 2002.

Fu, X., Harberd, N.P.: Auxin promotes Arabidopsis root growth by modulating gibberellin response. - Nature 421: 740, 2003.

Fukaki, H., Tasaka, M.: Hormone interactions during lateral root formation. - Plant mol. Biol. 69: 437, 2009.

Gou, J., Strauss, S. H., Tsai, C.J., Fang, K., Chen, Y., Jiang, X., Busov, V.B.: Gibberellins regulate lateral root formation in Populus through interactions with auxin and other hormones. - Plant Cell 22: 623-639, 2010.

Han, Y., Gao, Y., Shi, Y., Du, J., Zheng, D., Liu, G.: Genomewide transcriptome profiling reveals the mechanism of the effects of uniconazole on root development in Glycine max. - J. Plant Biol. 60: 387-403, 2017.

Jung, K.W., Oh, S.I., Kim, Y.Y., Yoo, K.S., Cui, M.H., Shin, J.S.: Arabidopsis histidine-containing phosphotransfer factor 4 (AHP4) negatively regulates secondary wall thickening of the anther endothecium during flowering. - Mol. Cells 25: 294300, 2008.

Katja, G., Maik, B.H.: Dynamic subnuclear relocalization of WRKY40, a potential new mechanism of ABA-dependent transcription factor regulation. - Plant Signal Behav. 10: e1106659, 2015.

Kim, D., Pertea, G., Trapnell, C., Pimentel, H., Kelley, R. Salzberg, S.L.: TopHat2: accurate alignment of transcriptomes in the presence of insertions, deletions and gene fusions. Genome Biol. 14: R36, 2013.

Li, H. (ed.): Botanical Microtechnique. - Science Press, Beijing 2009.

Mauriat, M, Petterle, A., Bellini, C., Moritz, T.: Gibberellins inhibit adventitious rooting in hybrid aspen and Arabidopsis by affecting auxin transport. - Plant J. 78: 372-384, 2014.

Manoharlal, R., Saiprasad, G.V.S., Thambrahalli, A., Madhavakrishna K.: Dissecting the transcriptional networks underlying the gibberellin response in Nicotiana tabacum. Biol Plant. 62: 647, 2018.

Noh, S.A., Choi, Y.I., Cho, J.S., Lee, H.: The poplar basic helix-loop-helix transcription factor BEE3-Like gene affects biomass production by enhancing proliferation of xylem cells in poplar. - Biochem. biophys. Re.s Commun. 462: 64-70, 2015.

Ogawa, M., Hanada, A., Yamauchi, Y., Kuwahara, A., Kamiya, Y. Yamaguchi, S.: Gibberellin biosynthesis and response during Arabidopsis seed germination. - Plant Cell 15: 1591-1604, 2003.

Osmont, K. S., Sibout, R., Hardtke, C.S.: Hidden branches: developments in root system architecture. - Annu. Rev. Plant Biol. 58: 93-113, 2007.

Peng, J.: The role of GA-mediated signalling in the control of seed germination. - Curr. Opin. Plant Biol. 5: 376-381, 2002.

Radwan, O., Liu, Y., Clough, S. J. Transcriptional analysis of soybean root response to Fusarium virguliforme, the causal agent of sudden death syndrome. - Mol. Plant Microbe Interact. 24: 958-972, 2011.

Rao, X., Huang, X., Zhou, Z., LIn, X.: An improvement of the $2^{-\Delta \Delta \mathrm{Ct}}$ method for quantitative real-time polymerase chain reaction data analysis. - Biostat. Bioinform. Biomath 3: 7185, 2013.

Ren, C., Kermode, A.R.: An increase in pectin methyl esterase 
activity accompanies dormancy breakage and germination of yellow cedar seeds. - Plant Physiol. 124: 231-242, 2000.

Schomburg, F.M., Bizzell, C.M., Lee, D.J., Zeevaart, J.A., Amasino, R.M.: Overexpression of a novel class of gibberellin 2-oxidases decreases gibberellin levels and creates dwarf plants. - Plant Cell 15: 151, 2003.

Slovak, R., Ogura, T., Satbhai, S.B., Ristova, D., Busch, W.: Genetic control of root growth: from genes to networks. Ann. Bot. 117: 9, 2015.

Smoot, M.E., Ono, K., Ruscheinski, J., Wang, P.L., Ideker, T.: Cytoscape 2.8: new features for data integration and network visualization. - Bioinformatics 27: 431-432, 2011.

Song, L., Prince, S., Valliyodan, B., Joshi, T., Maldonado dos Santos, J.V., Wang, J., Lin, L., Wan, J., Wang, Y., Xu, D., Nguyen, H.T.: Genome-wide transcriptome analysis of soybean primary root under varying water-deficit conditions. - BMC Genomics 17: 57, 2016.

Stamm, P., Topham, A.T., Mukhtar, N.K., Jackson, M.D., Tomé, D.F., Beynon, J.L., Bassel, G.W.: The transcription factor ATHB5 affects GA-mediated plasticity in hypocotyl cell growth during seed germination. - Plant Physiol. 173: 907, 2017.

Takase, T., Nakazawa, M., Ishikawa, A., Kawashima, M., Ichikawa, T., Takahashi, N., Shimada, H., Manabe, K., Matsui, M.: $y d k 1-D$, an auxin-responsive GA3 mutant that is involved in hypocotyl and root elongation. - Plant J. 37: 471-483, 2004.

Tanimoto, E.: Tall or short? Slender or thick? A plant strategy for regulating elongation growth of roots by low concentrations of gibberellin. - Ann. Bot. 110: 373-381, 2012.

Ubeda-Tomás, S., Federici, F., Casimiro, I., Beemster, G. T., Bhalerao, R., Swarup, R., Doerner, P., Haseloff, J., Bennett, M. J.: Gibberellin signaling in the endodermis controls Arabidopsis root meristem size. - Curr. Biol. 19: 1194-1199, 2009.

Ueguchi-Tanaka, M., Nakajima, M., Katoh, E., Ohmiya, H., Asano, K., Saji, S., Hongyu, X., Ashikari, M., Kitano, H., Yamaguchi, I. Matsuoka, M.: Molecular interactions of a soluble gibberellin receptor, GID1, with a rice DELLA protein, SLR1, and gibberellin. - Plant Cell 19: 2140-2155, 2007.

Wang, G.L., Que, F., Xu, Z.S., Wang, F., Xiong, A.S.: Exogenous gibberellin altered morphology, anatomic and transcriptional regulatory networks of hormones in carrot root and shoot. BMC Plant Biol. 15: 290, 2015.

Yuan, L.B., Dai, Y.S., Xie, L.J., Yu, L.J., Zhou, Y., Lai, Y.X., Yang, Y.C., Xu, L., Chen, Q.F., Xiao, S.: Jasmonate regulates plant responses to postsubmergence reoxygenation through transcriptional activation of antioxidant synthesis. - Plant Physiol. 173: 1864-1880, 2017. 